\title{
BOHR COMPACTIFICATION AND CONTINUOUS MEASURES
}

\author{
SADAHIRO SAEKI
}

ABsTrACT. Let $G$ be an LCA group with dual $\Gamma$. As a consequence of our main result, it is shown that every continuous regular measure $\mu$ concentrated on a Kronecker set and with norm $>1$ has the property that $\{|\hat{\mu}|>1\}$ is dense in the Bohr compactification of $\Gamma$.

Y. Katznelson [3] constructs a continuous measure $\mu$ on the circle group such that $\{n \in \mathbf{Z}:|\hat{\mu}(n)|>1\}$ is dense in the Bohr compactification of $\mathbf{Z}$. In the present note we point out that every continuous measure (with norm $>1$ ) concentrated on a Kronecker set has this property.

Let $G$ be a nondiscrete LCA group with dual $\Gamma$, and let $M(G)$ be the convolution measure algebra of $G$ (cf. [2]). For $\mu \in M(G)$, we denote by $\hat{\mu}$ the Fourier-Stieltjes transform of $\mu$. It is easy to show that every measure $\mu \in M(G)$ concentrated on a Kronecker set (or a $K_{p}$-set) has the following property, which we call (HK): Given $\gamma \in \Gamma$, a Borel set $E$ in $G$, and $\varepsilon>0$, there exists $\chi \in \Gamma$ such that

$$
\int_{E}|\gamma-\chi| d|\mu|+\int_{G \backslash E}|1-\chi| d|\mu|<\varepsilon
$$

TheOREM. Let $\mu \in M(G)$ be a continuous measure having property $(H K)$, and let $\mu_{1}, \ldots, \mu_{n} \in M(G)$ be absolutely continuous with respect to $\mu$. Then, for each nonempty (relatively) open subset $U$ of $\left\{\left(\hat{\mu}_{1}(\gamma), \ldots, \hat{\mu}_{n}(\gamma)\right): \gamma \in \Gamma\right\} \subset \mathbf{C}^{n}$, the set

$$
\left\{\gamma \in \Gamma:\left(\hat{\mu}_{1}(\gamma), \ldots, \hat{\mu}_{n}(\gamma)\right) \in U\right\}
$$

is dense in the Bohr compactification of $\Gamma$.

To prove this, we need two lemmas. Let $\mathbf{T}=\{z \in \mathbf{C}:|z|=1\}$ be the circle group.

LEMMA 1. Given $\varepsilon>0$, there exists a natural number $r$ having the following property. If $z_{1}, z_{2}, \ldots, z_{N}$ are finitely many elements of $\mathbf{T}$, there exist $k(1)$, $k(2), \ldots, k(r) \in\{1,2, \ldots, N\}$ and $p(1), p(2), \ldots, p(r) \in \mathbf{Z}$ such that

$$
\left|z_{1} \cdots z_{N}-z_{k(1)}^{p(1)} \cdots z_{k(r)}^{p(r)}\right|<\varepsilon .
$$

Proof. Let $S$ be the subgroup of $\mathbf{T}$ that is generated by all elements of $\mathbf{T}$ having order less than $2 \pi / \varepsilon$. Then $S$ is a finite group. We define $r$ to be the order of $S$.

Received by the editors September 26, 1979.

AMS (MOS) subject classifications (1970). Primary 43A25; Secondary 43A45.

Key words and phrases. Bohr compactification, continuous measure, Kronecker set, $K_{p}$-set. 
Now let $z_{1}, \ldots, z_{N} \in \mathbf{T}$ be given. If $\operatorname{ord}\left(z_{j}\right)>2 \pi / \varepsilon$ for some index $j$, then $\left\{z_{j}^{p}\right.$ : $p \in \mathbf{Z}\}$ is $\varepsilon$-dense in $\mathbf{T}$. Therefore it sufficies to set $k(1)=k(2)=\cdots=k(r)=j$, $p(1)=p$ for some $p \in Z$, and $p(2)=\cdots=p(r)=0$. So assume that $\operatorname{ord}\left(z_{k}\right)<$ $2 \pi / \varepsilon$ for all indices $k$. Then $\left\{z_{k}: 1 \leqslant k \leqslant N\right\}$ is contained in $S$ and therefore consists of at most $r$ different elements. Evidently this completes the proof.

LEMMA 2. Suppose $\rho \in M(G)$ is a nonnegative continuous measure having property $(H K), F$ is a finite set in $G, \gamma_{0}, \gamma_{1} \in \Gamma$ and $\varepsilon>0$. Then there exists $\gamma_{2} \in \Gamma$ such that

$$
\left|\gamma_{0}-\gamma_{2}\right|<\varepsilon \text { on } F \text { and } \int\left|\gamma_{1}-\gamma_{2}\right| d \rho<\varepsilon \text {. }
$$

Proof. Replacing $\gamma_{1}$ by $\bar{\gamma}_{0} \gamma_{1}$, we may assume that $\gamma_{0}=1$. Let us enumerate the elements of $F$ as $x_{1}, \ldots, x_{n}$, and define $F_{0}=\varnothing$ and $F_{j}=\left\{x_{1}, \ldots, x_{j}\right\}$ for $j=1$, $2, \ldots, n$. Put $\nu=\delta_{1}+\cdots+\delta_{n}+\rho$, where $\delta_{j}$ is the unit point measure at $x_{j}$. For $\mu \in M(G)$, we denote by $\Gamma(\mu)$ the closure of $\Gamma$ in $L^{1}(\mu)$. Notice that $\Gamma(\mu)$ forms a multiplicative group.

By induction on $j=0,1, \ldots, n$, we shall prove that given $f \in \Gamma(\rho)$, there exists $g \in \Gamma(\nu)$ such that $g=f$ a.e. $d \rho$ and $g=1$ on $F_{j}$. Since $F$ is a finite set and $F_{0}=\varnothing$, this is obvious for $j=0$. So assume that $1<j \leqslant n$ and that the result is true for $j-1$. Choose and fix any $f \in \Gamma(\rho)$ and any $\varepsilon>0$.

Let $r=r(\varepsilon)$ be the natural number given by Lemma 1. Since $\rho$ is a continuous measure, we can partition $\Gamma$ into disjoint Borel sets $E_{1}, E_{2}, \ldots, E_{N}$ such that $\rho\left(E_{k}\right)<\varepsilon /(2 r)$ for all $k=1,2, \ldots, N$. Without loss of generality, assume that $N>r$. Since $\rho$ has property (HK) and $f \in \Gamma(\rho)$, it is obvious that there exist $f_{1}$, $f_{2}, \ldots, f_{N}$ in $\Gamma(\rho)$ such that $f_{k}=f$ a.e. $d \rho$ on $E_{k}$, and $f_{k}=1$ a.e. $d \rho$ on $G \backslash E_{k}$. It follows from the inductive hypothesis that there exist $g_{1}, g_{2}, \ldots, g_{N}$ in $\Gamma(\nu)$ such that $g_{k}=f$ a.e. $d \rho$ on $E_{k}, g_{k}=1$ a.e. $d \rho$ on $G \backslash E_{k}$, and $g_{k}=1$ on $F_{j-1}$. We now apply Lemma 1 with $z_{k}=g_{k}\left(x_{j}\right)$ to find $k(1), \ldots, k(r) \in\{1,2, \ldots, N\}$ and $p(1), \ldots, p(r) \in \mathbf{Z}$ such that

$$
\left|\prod_{k=1}^{N} g_{k}\left(x_{j}\right)-\prod_{i=1}^{r}\left\{g_{k(i)}\left(x_{j}\right)\right\}^{p(i)}\right|<\varepsilon .
$$

There is no loss of generality in assuming that $k(i)=i$ for all $i=1, \ldots, r$. Define $h=\left(g_{1} \cdots g_{N}\right) /\left(g_{1}^{p(1)} \cdots g_{r}^{p(r)}\right)$. Then $h$ in an element of $\Gamma(\nu), h=1$ on $F_{j-1}$, $\left|h\left(x_{j}\right)-1\right|<\varepsilon$, and

$$
\int|h-f| d \rho=\sum_{k=1}^{r} \int_{E_{k}}|h-f| d \rho \leqslant 2 \sum_{k=1}^{r} \rho\left(E_{k}\right)<\varepsilon .
$$

Consequently we have proved that there exists a sequence $\left(h_{m}\right)$ in $\Gamma(\nu)$ such that $\left|h_{m}-1\right|<1 / m$ on $F_{j}$ and $\int\left|h_{m}-f\right| d \rho<1 / m$ for all $m>1$. Noting that $F$ is a finite set and passing to a subsequence, we may assume that $\left(h_{m}\right)$ converges to an element $g \in \Gamma(\nu)$. It is obvious that $g=1$ on $F_{j}$ and $g=f$ a.e. $d \rho$, which establishes our induction.

Finally we apply the above result for $j=n$ and $f=\gamma_{1}$. Thus there exists $g \in \Gamma(\nu)$ such that $g=\gamma_{1}$ a.e. $d \rho$ and $g=1$ on $F_{n}=F$. Since $\Gamma$ is dense in $\Gamma(\nu)$, this completes the proof. 
Proof of the Theorem. Let $\mu, \mu_{1}, \ldots, \mu_{n} \in M(G)$ and $U$ be as in the hypotheses of the Theorem. Let $b(\Gamma)$ denote the Bohr compactification of $\Gamma$ and let $\chi \in b(\Gamma)$ be given. We must prove that $\chi$ belongs to the closure of $\{\gamma \in \Gamma$ : $\left.\left(\hat{\mu}_{1}(\gamma), \ldots, \hat{\mu}_{n}(\gamma)\right) \in U\right\}$ in $b(\Gamma)$.

To this end, choose any finite subset $F$ of $G$ and any $\eta>0$. Then there exists $\gamma_{0}$ in $\Gamma$ such that $\left|\chi-\gamma_{0}\right|<\eta / 2$ on $F$. Let $\gamma_{1} \in \Gamma$ and $\varepsilon>0$ be such that

$$
\left\{\left(\hat{\mu}_{1}(\gamma), \ldots, \hat{\mu}_{n}(\gamma)\right): \gamma \in \Gamma,\left|\hat{\mu}_{j}\left(\gamma_{1}\right)-\hat{\mu}_{j}(\gamma)\right|<\varepsilon \forall j\right\} \subset U .
$$

Now we define $\rho=\left|\mu_{1}\right|+\cdots+\left|\mu_{n}\right| \in M(G)$. Then $\rho$ is a nonnegative continuous measure and has property (HK), as is easily seen. It follows from Lemma 2 that there exists $\gamma_{2}$ in $\Gamma$ such that $\left|\gamma_{0}-\gamma_{2}\right|<\eta / 2$ on $F$ and $\int\left|\gamma_{1}-\gamma_{2}\right| d \rho<\varepsilon$. Then we have $\left|x-\gamma_{2}\right|<\left|\chi-\gamma_{0}\right|+\left|\gamma_{0}-\gamma_{2}\right|<\eta$ on $F$, and

$$
\left|\hat{\mu}_{j}\left(\gamma_{1}\right)-\hat{\mu}_{j}\left(\gamma_{2}\right)\right| \leqslant \int\left|\gamma_{1}-\gamma_{2}\right| d\left|\mu_{j}\right|<\int\left|\gamma_{1}-\gamma_{2}\right| d \rho<\varepsilon
$$

for all $j=1,2, \ldots, n$. Therefore $\left(\hat{\mu}_{1}\left(\gamma_{2}\right), \ldots, \hat{\mu}_{n}\left(\gamma_{2}\right)\right)$ is in $U$. Since $F$ was an arbitrary finite set in $G$ and $\eta>0$ was arbitrary, this implies that $\chi$ is in the closure of the set $\left\{\gamma \in \Gamma:\left(\hat{\mu}_{1}(\gamma), \ldots, \hat{\mu}_{n}(\gamma)\right) \in U\right\}$. The proof is complete.

In order to state a corollary to the Theorem, we let $q(G)$ denote the largest member of $\{2,3, \ldots, \infty\}$ such that every neighborhood of $0 \in G$ contains an element of order $q$. Let $D(G)=\{z \in C:|z|<1\}$ if $q(G)=\infty$, and let $D(G)$ be the convex hull of $\{\exp (2 \pi i k / q(G)): k \in \mathbf{Z}\}$ in the complex plane if $q(G)<\infty$.

COROLlaRY. There exists a family $\left\{\mu_{i}: 0 \leqslant t \leqslant 1\right\}$ of continuous probability measures in $M(G)$ such that whenever $U$ is a nonempty open subset of $D(G)^{[0,1]}$, then $\left\{\gamma \in \Gamma:\left(\hat{\mu}_{t}(\gamma)\right)_{t} \in U\right\}$ is dense in the Bohr compactification of $\Gamma$.

Proof. As is well known (cf. [1]), $G$ contains a compact perfect set $K$ which is either a Kronecker set (if $q(G)=\infty$ ) or a $K_{q(G)}$-set (if $q(G)<\infty$ ). Let $\left\{E_{t}\right.$ : $0<t \leqslant 1\}$ be any family of pairwise disjoint perfect subsets of $K$, and let $\mu_{t}$ be any continuous probability measure concentrated on $E_{t}(0<t<1)$. Then it is easy to show that $\left\{\left(\hat{\mu}_{t}(\gamma)\right)_{t}: \gamma \in \Gamma\right\}$ is dense in $D(G)^{[0,1]}$. Therefore the required result is an immediate consequence of the present theorem.

\section{REFERENCES}

1. E. Hewitt and S. Kakutani, $A$ class of multiplicative linear functionals on the measure algebra of $a$ locally compact abelian group, Illinois J. Math. 4 (1960), 553-574.

2. W. Rudin, Fourier analysis on groups, Interscience Tracts in Pure and Appl. Math., No. 12, Interscience, New York and London, 1962.

3. Y. Katznelson, Sequences of integers dense in the Bohr group, Proc. Roy. Inst. Tech. (Stockholm) (1973), 79-86.

Department of Mathematics, Tokyo Metropolttan University, Fukazawa, Setagaya, Tokyo 158, JAPAN 